\title{
Phase dynamics in graphene-based Josephson junctions in the presence of thermal and correlated fluctuations
}

\author{
Claudio Guarcello, ${ }^{1,2}$ Davide Valenti, ${ }^{1}$ and Bernardo Spagnolo ${ }^{1,2,3}$ \\ ${ }^{1}$ Group of Interdisciplinary Theoretical Physics Università di Palermo and CNISM, \\ Unità di Palermo Viale delle Scienze, Edificio 18, 90128 Palermo, Italy \\ ${ }^{2}$ Radiophysics Department, Lobachevsky State University, \\ 23 Gagarin Avenue, 603950 Nizhniy Novgorod, Russia \\ ${ }^{3}$ Istituto Nazionale di Fisica Nucleare, Sezione di Catania, Via S. Sofia 64, I-90123 Catania, Italy
}

(Dated: July 23, 2018)

\begin{abstract}
In this work we study by numerical methods the phase dynamics in ballistic graphene-based short Josephson junctions. The supercurrent through a graphene junction shows a non-sinusoidal phase-dependence, unlike a conventional junction ruled by the well-known d.c. Josephson relation. A superconductor-graphene-superconductor system exhibits superconductive quantum metastable states similar to those present in normal current-biased JJs. We explore the effects of thermal and correlated fluctuations on the escape time from these metastable states, when the system is stimulated by an oscillating bias current. As a first step, the analysis is carried out in the presence of an external Gaussian white noise source, which mimics the random fluctuations of the bias current. Varying the noise intensity, it is possible to analyze the behavior of the escape time from a superconductive metastable state in different temperature regimes. Noise induced phenomena, such as resonant activation and noise induced stability, are observed. The study is extended to the case of a coloured Gaussian noise source, analyzing how the escape time from the metastable state is affected by correlated random fluctuations for different values of the noise correlation time.
\end{abstract}

\section{INTRODUCTION}

The possibility of obtaining graphene $e^{1}$, by extraction of single layers from graphite, paved the way for a new generation of superconductive graphene-based devices. In particular, the evidence of proximity-induced superconductivity ${ }^{2,3}$, due to the one-atom thick nature of graphene, promoted the realization of superconductorgraphene-superconductor (SGS) structures. The refractoriness of graphene to the surface oxidation in natural environment favours the realization of highly transparent contacts with the superconductive electrodes. Furthermore, superconductivity in graphene, pure or doped, was predicted and explored $\underline{4,5}$ and new devices, as dcSQUIDs ${ }^{6.7}$, proximity Josephson sensors ${ }^{8}$, or bolometers based on superconductive tunnel junction contacts ${ }^{9}$, were fabricated using graphene. The charge carriers in graphene are massless quasiparticle, the Dirac fermions, with pseudo-spin half and linear energy dispersion ${ }^{10}$. The band structure shows contact points, called Dirac points,

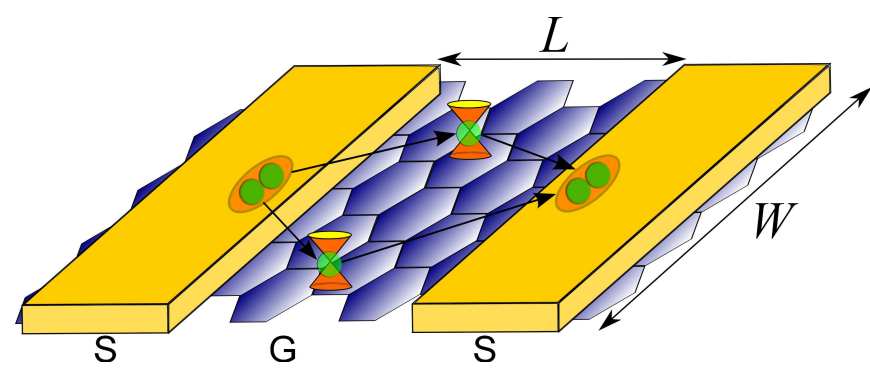

FIG. 1. (Color online) Schematic view of a suspended SGS device. The electrons forming a Cooper pair, when they enter graphene, move into different $\mathrm{K}$-valleys, represented as orange cones. In the short-junction regime, $L \ll W$. beetwen the conduction and valence bands ${ }^{10}$. These peculiar electronic properties ${ }^{10}$ give rise to interesting phenomena, such as specular Andreev reflection ${ }^{11}$, unusual propagating modes along graphene channels ${ }^{12}$, oscillatory dependence of the Josephson current on the barrier thickness and applied bias voltage $\frac{13}{}$. Titov and Beenakker ${ }^{14}$ predicted, in the limit of zero temperature, the behavior of critical current and current-phase relationship (C $\Phi \mathrm{R})$ for a short ballistic SGS system. Taking a cue from these results, Lambert et al $\stackrel{15}{15}$ showed the existence of a plasma frequency and a washboard potential for a suspended graphene junction.

A Josephson junction (JJ) is a mesoscopic system in which macroscopic quantities, as current and voltage, are directly dependent on the transient dynamics of a microscopic order parameter. The output of this device is strongly affected by environmental perturbations, that is stochastic fluctuations of temperature, current or magnetic field. Different aspects of graphene-based junctions in noisy environment were already examined by several authors. Miao et al $\stackrel{16}{n}$ took into account the noise induced premature switching in underdamped SGS JJ at finite temperature. Specifically, in Ref. [16] the reduction of the critical current and variations in the product $I_{C} R_{N}$ were experimentally observed and theoretically explained considering non-negligible the thermal fluctuations. Other authors ${ }^{3,17}$ suggested a supercurrent reduction by premature switching induced by thermal and electromagnetic noise. Coskun et al $\stackrel{18}{n}$ systematic studied the thermally activated dynamics of phase slip in SGS JJs throught the measurement of the switching current distribution. They found an anomalous temperature dependence of the switching current dispersion due to nontrivial structure ${ }^{14,19}$ of the Josephson current. A sim- 
ple stochastic model to explore the electrodynamics of an underdamped graphene JJ was proposed by Mizuno et $a l^{20}$. They stressed the importance of realizing high quality suspended SGS structures, to prevent disorders due to the conventionally used substrates, whereby a flow of supercurrent at high critical temperature can be obtained. The SGS junction is a good candidate for the fabrication of gate-tunable phase qubits ${ }^{21.22}$. In Ref. 21] the study of the stochastic switching current distribution in a SGS junction for low temperatures allowed to highlight the macroscopic quantum tunneling and energy level quantization, similarly to conventional JJs. Moreover, Lee et al $\stackrel{21}{\underline{n}}$ studied the switching current distribution in both quantum and thermal regime, building up a computational analysis based on the pure resistively and capacitively shunted junction (RCSJ) model for a conventional $\mathrm{JJ}^{23}$. Considering a range of temperatures in which the dynamics is exclusively ruled by thermal fluctuations, Lee et al ${ }^{21}$ observed disagreement beetwen the experimental and fitted temperatures. To understand this discrepancy, they invoked the misuse in the model of the pure sinusoidal Josephson current distribution, neglecting however any noise induced effects on the escape rate from the superconductive state.

Our work fits well into this assorted scenario, since it aims to study how thermal fluctuations affect the behavior of a SGS junction. In particular, we study the influence of Gaussian (white or colored) noise sources on the switching dynamics from the superconductive metastable state to the resistive one in a suspended graphene-based short $J J$, considering the proper $C \Phi \mathrm{R}^{14}$. We recall that the effects of thermal fluctuations on the dynamics of conventional short $\underline{24-28}$ and long $\underline{29}-\underline{33}$ JJs have been thoroughly investigated, both theoretically predicting $24-28,33,34$ and experimentally observing ${ }^{35-37}$ noise induced effects in the superconductive lifetime of a JJ. The rate of switching from the JJ metastable superconducting state encodes information about the noise present in an input sig-

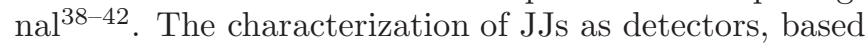
on the statistics of the escape times, has been recently proposed $\underline{38-44}$. Specifically, the statical analysis of the switching from the metastable superconducting state to the resistive running state of the JJ has been proposed to detect weak periodic signals embedded in a noisy environment $\underline{43.44}$. In this paper we explore therefore the transient dynamics of an underdamped SGS junction, considering the simultaneous action of an external driving force oscillating with frequency $\omega$, and a stochastic signal which represents a random force of intensity $\gamma$. We focus our analysis on the mean permanence time in the superconductive state. The study is performed fixing the initial values of the applied bias current $i_{0}$ and the correlation time $\tau_{c}$ of the colored noise source, and varying the frequency $\omega$ and the noise intensity $\gamma$. Whenever possible, we compare our results with results obatained for normal JJs. The similarities in the behavior of the graphene-based and normal junctions allow to interprete our results referring to conventional JJ quantities, such as plasma frequency (Eq. 4).

The paper is arranged as follows. The next section includes an overview about the physical model used. In Sec. III the statistical features of Gaussian and correlated noise sources are examined. Sec. IV contains the computational details. In Sec. V the theoretical results are shown and analyzed. The Sec.VI contains a probability density function (PDF) analysis of the escape times carried out setting the system parameters associated with the appearance of noise induced non monotonic effects in the mean switching times. In Sec. VII conclusions are drawn.

\section{THE MODEL}

The electrodynamics of a JJ can be explored looking at the time evolution of the order parameter $\varphi$, that is the phase difference between the wave functions of the two coupled superconductors forming the device. According to the RCSJ model and including the environmental influence, the equation of motion for $\varphi$ is

$$
\varphi_{t t}(t)+\beta_{J} \varphi_{t}(t)=i_{b}(t)-i_{\varphi}(t)+i_{f}(t)
$$

in which $i_{b}(t)$ and $i_{\varphi}(t)$ are the bias and supercurrent respectively, both normalized to the critical current of the junction $i_{c}$. The term $i_{f}(t)$ represents the stochastic noise contribution. The subscripts of $\varphi$ denote partial derivatives in time. The use of normalized variables allows to extend, in a direct and simple way, the theoretical results to different experimental settings. Eq. (1) is in accordance with the Johnson approach ${ }^{23}$, since it includes a damping parameter $\beta_{J}=\left(\omega_{p_{0}} R_{N} C\right)^{-1}$, multiplied by $\varphi_{t}(t)$, and assumes the time variable normalized to the inverse of the zero-bias plasma frequency $\omega_{p_{0}}=\sqrt{2 \pi i_{c} /\left(\Phi_{0} C\right)}\left(R_{N}\right.$ and $C$ are the normal resistance and capacitance of the junction, and $\Phi_{0}=h / 2 e$ is the magnetic flux quantum). Introducing the parameter $\beta_{C}=\beta_{J}{ }^{-2}$, Eq. (11) can be alternatively arranged in the Stewart-McCumber framework ${ }^{23}$, according which a term $\beta_{C} \varphi_{t t}(t)$ is included in the equation, and the time variable is normalized to the inverse of the $\mathrm{JJ}$ characteristic frequency $\omega_{c}=\omega_{p_{0}}^{2} R_{N} C$. The JJ behavior can be depicted as the motion of a "phase particle" with mass $m=C\left(\Phi_{0} / 2 \pi\right)^{2}$ rolling down along the profile of a potential, called the washboard potential, composed by a tilted sequence of wells. For a conventional current biased junction, the normalized supercurrent and washboard potential have the well-known expressions

$$
\begin{aligned}
i_{\varphi}(t) & =\sin (\varphi(t)) \\
U(\varphi, t) & =-E_{J 0}\left[\cos (\varphi(t))+i_{b}(t) \varphi(t)\right],
\end{aligned}
$$

where $E_{J 0}=\Phi_{0} i_{c} / 2 \pi$ is the Josephson coupling energy, that is the energy initially stored in the junction. The bias current represents the slope of this potential. Eq. (2) is the d.c. Josephson relation. In the limit of small amplitude oscillations, the JJ plasma frequency corresponds 


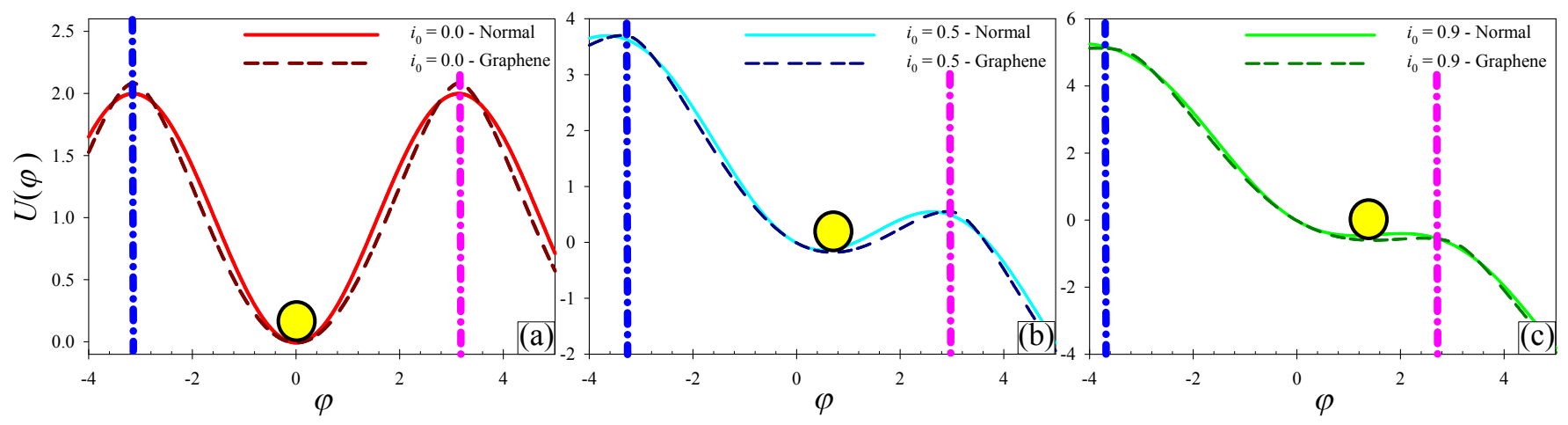

FIG. 2. (Color online) Washboard potential for conventional (see Eq. (3)) and graphene (see Eq. (9)) JJs (solid and dashed lines, respectively), for different initial values of the bias current: (a) $i_{0}=0.0$; (b) $i_{0}=0.5$; (c) $i_{0}=0.9$. It is also shown the initial position (bottom of the potential well) of the "phase particle". Blue and pink dotted-dashed lines indicate the left and right absorbing barriers, respectively.

to the oscillation frequency in the bottom of a potential well, modified by the presence of a bias current according to

$$
\omega_{P}(t)=\omega_{p_{0}} \sqrt[4]{1-i_{b}^{2}(t)}
$$

Titov and Beenakker ${ }^{14}$ calculated the C $\Phi R$ and critical current for a ballistic graphene-based junction at the Dirac point. They address the problem in the framework of the Dirac-Bogoliubov-de Gennes (DBdG) equation 11,45. Considering the Josephson current at zero temperature $\frac{46}{4}$

$$
I(\varphi)=-\frac{4 e}{\hbar} \frac{\mathrm{d}}{\mathrm{d} \varphi} \int_{0}^{\infty} d \varepsilon \sum_{n=0}^{\infty} \rho_{n}(\varepsilon, \varphi) \varepsilon
$$

supposing an "ideal" normal-metal-superconductor interface and taking infinite mass boundary conditions 47 they obtained the following expressions

$$
\begin{aligned}
i_{\varphi}(t) & =\frac{i(\varphi)}{i_{c}}=\frac{2}{1.33} \cos \left(\frac{\varphi}{2}\right) \tanh ^{-1}\left[\sin \left(\frac{\varphi}{2}\right)\right] \\
i_{c} & =1.33 \frac{e \Delta_{0}}{\hbar} \frac{W}{\pi L}
\end{aligned}
$$

where $W$ and $L$ are the linear dimensions of the device (see Fig. 1), that is the length of the superconductive plates and their separation, respectively. Furthermore $\Delta_{0}$ is the superconductive excitation gap, $e$ the electron charge and $\hbar$ the reduced Plank's constant. The Eqs. (6) and (7) refer to the short-junction regime, in which $L$ is smaller than the superconducting coherence length $\xi$, that is the distance to which a Cooper pair spreads, and for short and wide normal region, i.e. $L \ll W$. We recall that the simple C $\Phi R$ given in Eq. 6 is obtained in the limit of zero temperature. Hagimásy et al $\underline{19}$ calculated a more general formula for finite temperature and arbitrary junction length. However, an analytic expression for the Josephson current, such as that given in terms of washboard potential, can not be obtained except for
$T=0$. Indeed, for vanishing temperature the expression by Hagimásy et al. correctly converges to that obtained by Titov and Beenakker. Instead, for $T \rightarrow T_{c}$, the nonsinusoidal supercurrent derived by Hagimásy et al. in both long and short junction regime, converges to a sinusoidal behavior. In the short junction limit, cf. Fig. 1a and Fig. 3a in Ref. [19], as long as $T \lesssim T_{c} / 4$, the critical current and $i(\varphi)$ hardly change, so that Titov and Beenakker's formula remains valid ${ }^{48}$. This temperature threshold can be also deduced from the gap equation of the BCS theory, cf. Eq. (8) of Ref. [19]. The work presented in this paper is therefore strictly valid in a wide range of temperature values, and represents a good approximation for temperatures far from the critical value. For completeness, in the long junction limit, $L \gg W$, the Josephson current reduces to

$$
I_{\varphi}=\frac{e \Delta}{\hbar} \tanh \left(\frac{\Delta}{2 T}\right) e^{-\pi L / W} \sin \varphi=I_{c}(T) \sin \varphi
$$

showing the same $\varphi$-dependence of conventional JJs (see supplemental material of Ref. 18). Lambert et al. ${ }^{15}$ proposed, for the Titov's Josephson current, the following washboard-like potential

$$
\begin{aligned}
\tilde{U}(\varphi, t)= & -E_{J 0}\left\{-\frac{2}{1.33}\left\{2 \sin \left(\frac{\varphi}{2}\right) \tanh ^{-1}\left[\sin \left(\frac{\varphi}{2}\right)\right]+\right.\right. \\
& \left.\left.+\ln \left[1-\sin ^{2}\left(\frac{\varphi}{2}\right)\right]\right\}+i_{b}(t) \varphi\right\}
\end{aligned}
$$

The analytic knowledge of the potential allows to well impose the initial condition and the thresholds for the escape time calculations. As well as the conventional $U(\varphi, t)$ (see Eq. 3), the potential $\tilde{U}(\varphi, t)$ consists of a tilted sequence of wells. The position of the phase particle or, more precisely, its dynamical condition along this potential, defines the working regime of the junction. In the superconductive state the particle lies in a well, while in the resistive state it rolls down along the potential. 


\begin{tabular}{|c|c|c|c|c|c|c|}
\hline & $\begin{array}{c}\mathrm{Mizu}- \\
\mathrm{no} \underline{\underline{20}}\end{array}$ & Coskun 18 & $\mathrm{Du}^{3}$ & $\begin{array}{l}\text { Heer- } \\
\text { schee }^{2}\end{array}$ & $\begin{array}{c}\text { English }^{51} \\
\text { Samples } \\
\text { A/B/C/D }\end{array}$ & Miao $\frac{16}{n}$ \\
\hline $\begin{array}{ll}i_{C} & \mathrm{~mA}\end{array}$ & 100 & 10 & 800 & 10 & \begin{tabular}{|l|}
$71 / 107$ \\
$39 / 160$
\end{tabular} & 110 \\
\hline$\beta_{C}$ & 76 & 16 & & & & \\
\hline $\mathrm{pF}$ & 1 & $12-50$ & & & & \\
\hline $\begin{array}{ll}R_{N} & \Omega\end{array}$ & 500 & 10 & & & & \\
\hline $\bar{K}$ & 3 & 0.4 & 0.2 & 0.3 & 0.01 & 0.3 \\
\hline $\begin{array}{ll}T_{C O} & \mathrm{~K} \\
\end{array}$ & 0.02 & {$[8-17] \cdot 10^{-3}$} & & & & \begin{tabular}{|l|}
$0.12-1.2$ \\
\end{tabular} \\
\hline$\overline{\gamma_{C}}$ & 1.3 & 1.7 & 0.01 & 1.3 & $\begin{array}{c}6 / 4 / 11 / 3 \\
\cdot 10^{-3}\end{array}$ & \begin{tabular}{|l|}
0.11 \\
\end{tabular} \\
\hline$\omega_{P 0} \mathrm{GHz}$ & 17 & $0.8-1.6$ & & & & $10^{2}-10^{3}$ \\
\hline
\end{tabular}

TABLE I. Experimental values of different JJ parameters, calculated or directly acquired by various published works.

When this happens, a non-zero mean voltage $V$ across the junction appears, according to the a.c. Josephson relation, $\varphi_{t}=2 \pi V / \Phi_{0}$. Furthermore, depending on the damping parameter value, the phase diffusion state, that is an escape event with a retrapping in the first subsequent minimum, could be established. When $i_{b}(t) \geq 1$, that is when the applied bias current exceeds the critical value, both potentials (Eqs. (3) and (90) lose their "maxima and minima" structures and the particle tends to freely slip.

We explore the response of the system to the simultaneous action of both d.c. and a.c. current sources. The bias current, composed by a constant term, $i_{0}$, representing its initial value, and an oscillating part whose frequency $\omega$ is normalized to $\omega_{p_{0}}$, is therefore given by

$$
i_{b}(t)=i_{0}+A \sin (\omega t) .
$$

By choosing properly the values of $i_{0}$ and $A$, within a period it is possible to achieve values of $i_{b}(t)$ greater than 1. A direct comparison between the potentials for normal and graphene-based JJ is given in Fig. 2 for $i_{0}=$ 0.0 (panel a), 0.5 (panel b), 0.9 (panel c). Here it is worth noting that differences, though small, between the graphene and normal JJ curves are detectable. Fig. 2 shows also the initial condition for the fictitious particle, which is located in the potential minimum. We can assume that the system leaves the superconductive regime when the particle reaches one of the nearest maxima. Two absorbing barriers are therefore placed in correspondence to these maxima, as highlighted in Fig. 2 (see dotted-dashed lines). Recording for each realization the escape times $t^{e s c}$, i.e. the time required to pass a barrier, for an enough large number $N$ of realizations, the mean first passage time (MFPT) is defined as

$$
\tau=\frac{1}{N} \sum_{i=1}^{N} t_{i}^{e s c} .
$$

The oscillating force acting on the system, $i_{b}(t)$, and stochastic fluctuations, $i_{f}(t)$, due to the environmental influence, drive the switching dynamics. Two different mechanisms can therefore cause overcome of the potential barrier: the macroscopic quantum tunneling or the thermally activated passage. These processes are triggered in distinct ranges of temperature so that, for vanishing values of the bias and damping, a threshold value exists, $T_{C O}=\hbar \omega_{p_{0}} / 2 \pi k$ ( $k$ is the Boltzmann constant), called crossover temperature. In a damped system, when a polarization current is applied, this value is slightly reduced, becoming $\underline{49}$ :

$$
T_{C O}^{\star}=\hbar \omega_{R} / 2 \pi k,
$$

where $\omega_{R}=\omega_{P}\left\{\sqrt{1+\alpha^{2}}-\alpha\right\}, \alpha=\left(2 \omega_{P} R_{N} C\right)^{-1} \propto$ $\beta_{J}$. For $T<T_{C O}^{\star}$ the system undergoes a quantum tunneling regime. On the other hand, for $T>T_{C O}^{\star}$, the system works in the thermal activation regime. Here we do not take into account quantum effects. In this condition, when thermal fluctuations are neglected, the phase can remarkably change merely as the applied current approaches the critical value $i_{c}$ (the system moves into a resistive regime). Conversely, considering noise effects, transitions along the potential can occur also applying a current much smaller than $i_{c}$. As already pointed out, the phase dynamics is affected by dissipative phenomena, responsible for peculiarities of the system, ranging from overdamped (high viscosity $\beta_{J} \gg 1$ ) to underdamped (low viscosity $\left.\beta_{J} \ll 1\right)$ condition. The Table \ shows a collection of few experimental values, for different graphene-based JJs, calculated or, whenever possible, directly acquired by different published works, 2, 16,18,20,51. Blank cells indicate that the value of the related variables are not available. The values of the parameters $\beta_{C}=\beta_{J}{ }^{-2}$ suggest that these systems often $\frac{18,20}{}$ work in underdamped conditions. Moreover, the comparison between the working temperature $T$ and the crossover value $T_{C O}^{\star}$, underlines the thermally activated behavior of the switching dynamics characterizing these junctions $16,18,20$.

The noise source. - An exhaustive analysis of a real device has to take into account environmental fluctuations continuously affecting the system, such as unpredictable variations of current and temperature. Thus the deterministic RCSJ model can be improved by considering the presence of the stochastic current $i_{f}$ (see Eq.(1), in a first approximation modeled using a Gaussian "white" noise source. The stochastic non-normalized current $I_{f}(\tilde{t})$ is therefore characterized by the well-known statistical properties of a Gaussian random process

$$
\left\langle I_{f}(\tilde{t})\right\rangle=0 \quad\left\langle I_{f}(\tilde{t}) I_{f}(\tilde{t}+\tilde{\tau})\right\rangle=2 \frac{k T}{R_{N}} \delta(\tilde{\tau}),
$$

where $T$ is the temperature. Using normalized current and time, the correlation function becomes

$$
\left\langle i_{f}(t) i_{f}(t+\tau)\right\rangle=2 \gamma(T) \delta(\tau),
$$

where the dimensionless amplitude $\gamma(T)$ is proportional to the temperature $T$. We note that the expression of 
$\gamma(T)$ depends on the approach used to manage Eq. (11)

$$
\begin{aligned}
\text { McCumber) } & \gamma^{c}(T) & =\frac{k T}{R_{N}} \frac{\omega_{c}}{i_{c}^{2}}=\frac{2 e}{\hbar} \frac{k T}{i_{c}}=\frac{k T}{E_{J}} \\
\text { Johnson) } & \gamma^{p}(T) & =\frac{\omega_{p_{0}}}{\omega_{c}} \gamma^{c}(T)
\end{aligned}
$$

It is worth noting that the noise intensity can be also expressed as the ratio between the thermal and Josephson coupling energies (see Eq. (15a)). Few $\gamma^{c}$ values, calculated for several experimental setting, are shown in the Table I More in general, $i_{f}(t)$ can represent a Gaussian colored noise, modeled as an exponentially correlated noise source. Specifically, in this work the noise source is described by the well-known Ornstein-Uhlenbeck (OU) process 50

$$
d i_{f}(t)=-\frac{1}{\tau_{c}} i_{f}(t) d t+\frac{\sqrt{\gamma}}{\tau_{c}} d W(t),
$$

where $\gamma$ and $\tau_{c}$ are the intensity and correlation time of the noise source, respectively, and $W(t)$ is the Wiener process, characterized by the well-known statistical properties: $\langle d W(t)\rangle=0$ and $\left\langle d W(t) d W\left(t^{\prime}\right)\right\rangle=\delta\left(t-t^{\prime}\right) d t$.

The correlation function of the OU process is

$$
\left\langle i_{f}(t) i_{f}\left(t^{\prime}\right)\right\rangle=\frac{\gamma}{2 \tau_{c}} e^{-\frac{\left|t-t^{\prime}\right|}{\tau_{c}}},
$$

and gives $\gamma \delta\left(t-t^{\prime}\right)$ in the limit $\tau_{c} \rightarrow 0$.

Computational details. - The stochastic dynamics of the system is explored integrating Eqs. (10) and (16) by a finite difference method. Specifically, the stochastic differential equation (16) is integrated within the Ito scheme. The time step is fixed at $\Delta t=10^{-3}$ and the maximum time, for which equations are integrated, is $t_{\max }=100$, i.e. a time large enough to catch every nonmonotonic behavior. A collection of first passage times is obtained iterating the procedure for a sufficiently large number of realizations $N=10^{4}$. The initial condition to solve Eq. (11) is set at the bottom of a valley of the potential given in Eq. (9), closer to $\varphi=0$. During the oscillation of the potential the two absorbing barriers change their position, following the displacements of the neighboring maxima. The analysis is performed in the underdamped regime, setting $\beta_{J}=0.1$ (corresponding to $\beta_{C}=100$ ). Four different values of $i_{0}$, in the range $0 \leq i_{0}<1$, are used. The time periodical component of $i_{b}(t)$, oscillates with values of the frequency $\omega$ ranging within the interval $[0.01-10]$. In our analysis the intensity $\gamma$ of the colored noise source $i_{f}(t)$ varies in the range $\left[10^{-4}-10^{2}\right]$, with the correlation time, $\tau_{c}$, set at different values.

\section{THE ANALYSIS}

The analysis is performed studying the behavior of the MFPT, $\tau$, as a function of the noise intensity $\gamma$ and frequency $\omega$ of the oscillating term in the bias current. In
Eq. (10) $i_{0}=0.0,0.1,0.5,0.9$, corresponding to vanishing, small, intermediate and high values, respectively, of the initial slope of the washboard potential. The slope of the potential, that is the value of $i_{b}(t)$, is directly related to the height of the potential barriers, so that, increasing the value of $i_{b}(t)$, the right barrier's height decreases, getting zero when $i_{b}(t) \geq 1$. The normalized amplitude of the oscillating term of the bias current is set at $A=0.7$ in all numerical realizations.

We show the values of $\tau$ in three-dimensional plots to highlight the simultaneous presence of different nonmonotonic effects. The values of $\gamma$ are proportional, through Eqs. (15), to the temperature of the system, so that varying the noise intensity in the interval $\gamma=$ $\left[10^{-4}-10^{2}\right]$ corresponds to explore a wide range of temperatures. The noise amplitude values calculated in different frameworks and presented in the Table I] fall within this range. The values of the frequency $\omega$ are chosen in such a way to investigate different regimes of alternate current: i) quasi-direct current $(\omega \ll 1)$; ii) high-frequency alternate current $(\omega \gg 1)$; iii) alternate current oscillating at the characteristic plasma frequency of a conventional junction $(\omega=1)$. Recalling that the driving frequency is normalized to the plasma frequency, the values of $\omega_{P 0}$ included in Table $\square$ makes it possible to give a quantitative estimation to the values taken by $\omega$. The correlation time of the colored noise source takes the values $\tau_{c}=0$ (i.e. white noise), $1,5,10$. The results, shown in Fig. 3, were obtained using a white noise source, that is setting $\tau_{c}=0.0$, and for different values of the initial bias current (slope of the potential), i.e. $i_{0}$ $=0.0$ (panel a), 0.1 (panel b), 0.5 (panel c), 0.9 (panel d). First we can note that an overall lowering of $\tau$ values occurs, as $i_{0}$ increases. In other words, changes in the maximum slope of the potential cause modifications in the height of the barriers (see Fig. 2). The presence of two absorbing barriers allows to take into account the complete evolution of the phase particle from the initial state. Considering highly tilted potential profile (panel c of Fig. (2), the particle rolls down exclusively overcoming the right barrier. Instead, with small value of the initial bias current (panel a of Fig. 2), the possibility of escaping over the left-side barrier causes interesting phenomena. In particular, for $i_{0}=0.0$, the height of the left and right barriers takes on the same values within an oscillation period, so that the particle can escape through the left or right barrier with equal probability. In all panels of Fig. (3) it is evident a nonmonotonic behaviour, characterized by a minimum, which indicates the presence of a resonant activation (RA) phenomenon ${ }^{52-61}$. This effect is robust enough to be detected in a large range of $\gamma$ values, even if it tends to be suppressed (the minimum in the curves of MFPT $v s \omega$ is less pronounced) as the intensity, $\gamma$, of thermal fluctuations increases. In particular, two different kinds of RA can be distinguished: i) the dynamic resonant activation, which occurs as the driving frequency approaches the natural characteristic frequency of the system, coinciding for a JJ with the 

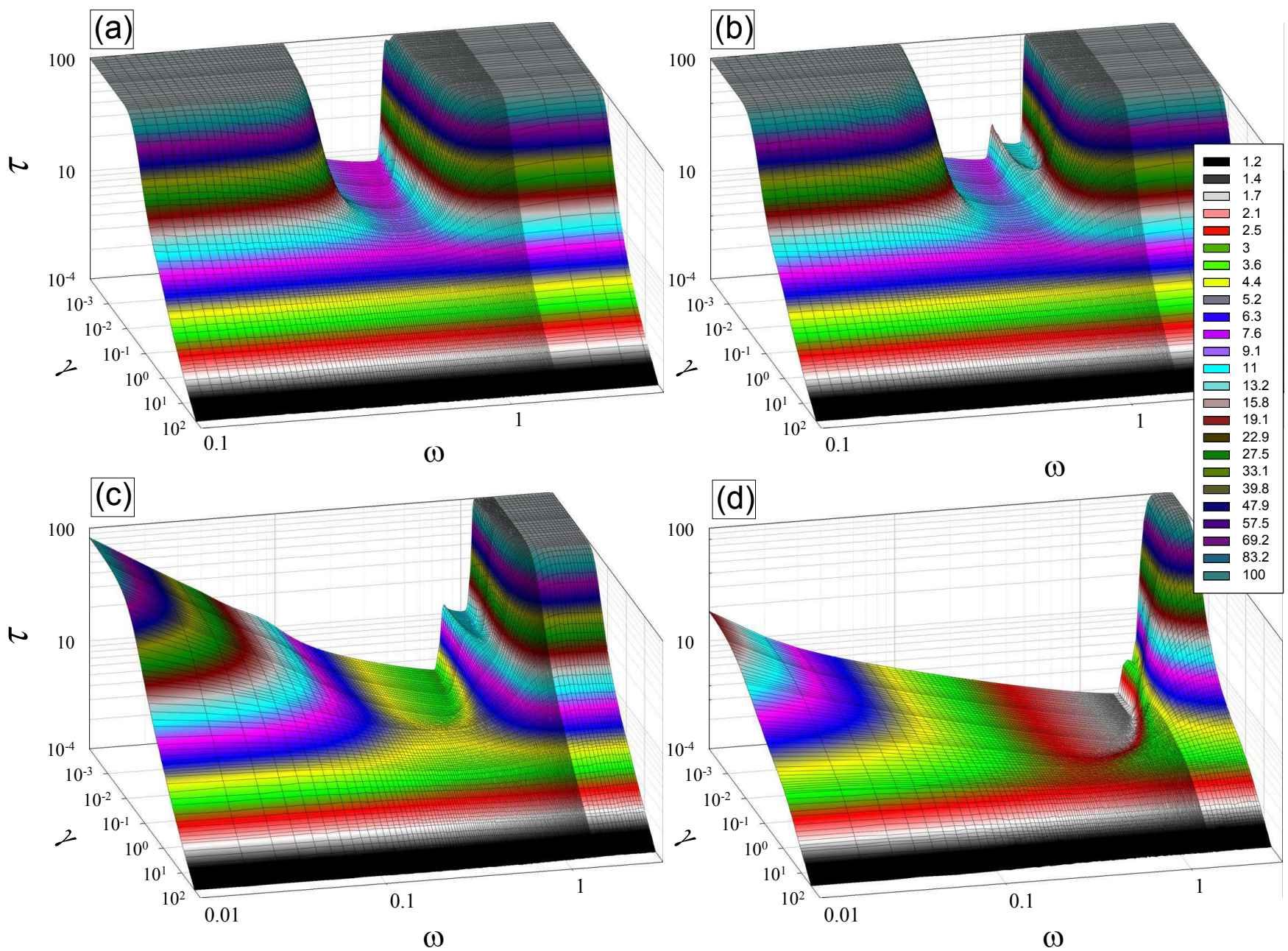

FIG. 3. (Color online) MFPT as a function of both $\omega$ and $\gamma$, for $\tau_{c}=0.0$ and different initial values of the bias current: (a) $i_{0}=0.0$ (no slope); (b) $i_{0}=0.1$ (small slope); (c) $i_{0}=0.5$ (intermediate slope); (d) $i_{0}=0.9$ (high slope). The legend in panel d refers to all pictures.

plasma frequency $\frac{62-64}{-6}$; ii) the stochastic resonant activation, which occurs for driving frequency close to the inverse of the average escape time at the minimum, i.e. the mean escape time over the potential barrier in the lowest configuration 37,44 . The dynamic RA is evident only in quasi-deterministic regime, i.e. $\gamma \ll 1$, when the dynamics depends mainly by the geometry and symmetry of the system. Increasing the noise intensity, the stochastic RA tends to overcome every dynamic RA effect. Fig. 4 shows the behaviour of the MFPT vs $\omega$, with the noise intensity fixed at such a value $\left(\gamma=10^{-4}\right)$ that the dynamic RA effect can be clearly observed and studied as a function of the initial bias current $i_{0}$. More in detail, Fig. 4 displays results obtained for graphene-based (solid lines) and normal (dotted lines) JJs. The MFPT values in correspondence of the RA minima are almost junctiontype independent, even if the RA valleys for normal JJ are shifted towards higher frequencies. In particular, the dynamic RA minima for small $i_{0}$ become narrower passing from SGS to normal junction. In Fig. 3, where the noise intensity is set at $\gamma=10^{-4}$, for $i_{0}=0.0$ (panel a) a single-minimum dynamic RA is present in $\omega_{d R A}^{0.0} \simeq 0.81$. The RA effect becomes more structured, slightly increasing the initial bias current. Indeed, for $i_{0}=0.1$ the same effect occurs with the presence of two minima located at $\omega_{d R A}^{0.1} \simeq 0.75,0.95$ (see panel b of Fig. 3). These minima are connected with two resonance phenomena occurring in the system. Specifically, the oscillating potential can "tune" with the plasma oscillations for two different values of $\omega$, one corresponding to escape events towards left, which occur at the lowest slope, the other one corresponding to escape events towards right, which occur at the highest slope. This double-resonance effect can be further explained, noting that non-vanishing values of the bias current $\left(i_{0} \neq 0\right)$ introduce an asymmetry, e.g. with $i_{0}=0.1$ the highest and lowest slope are respectively $\left|i_{b}\left(\mathrm{~T}_{p} / 4\right)\right|=0.8$ and $\left|i_{b}\left(3 \mathrm{~T}_{p} / 4\right)\right|=0.6$, where $\mathrm{T}_{p}$ is the oscillation period. For these configurations the plasma frequencies, calculated according to Eq. (4), are $\omega_{P}^{0.1}\left(\mathrm{~T}_{p} / 4\right) \simeq 0.77$ and $\omega_{P}^{0.1}\left(3 \mathrm{~T}_{p} / 4\right) \simeq 0.90$. These val- 


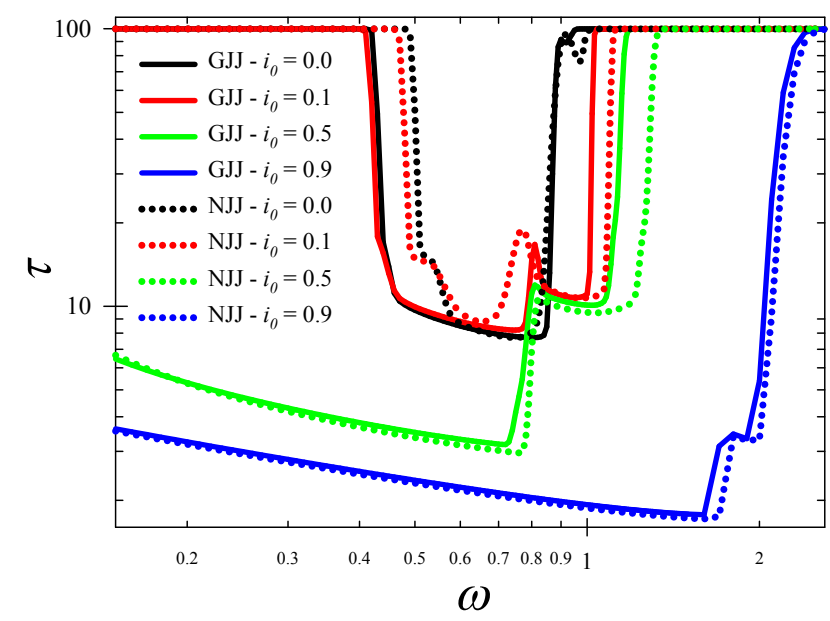

FIG. 4. (Color online) MFPT as a function of $\omega$, for $\gamma=$ $10^{-4}, \tau_{c}=0.0$, and different initial values of the bias current: $i_{0}=0.0,0.1,0.5,0.9$. Solid and dotted lines represent results for a graphene-based JJ (indicated as GJJ) and a normal JJ (indicated as NJJ), respectively.

ues, even if they do not coincide, are very close to the frequencies $\omega_{d R A}^{0.1}$ for which the RA minima are observed. The small discrepancies between $\omega_{d R A}^{0.1}$ and the two frequencies $\omega_{P}^{0.1}\left(\mathrm{~T}_{p} / 4\right)$ and $\omega_{P}^{0.1}\left(3 \mathrm{~T}_{p} / 4\right)$ can be related to the fact that the conventional JJ plasma frequency was used. Due to the symmetry of the potential for $i_{0}=0.0$ respect to the horizontal position, the minima observed for $i_{0}=0.1$ seem to merge in the larger minimum located, for vanishing bias current, at $\omega_{d R A}^{0.0} \simeq 0.81$ (see Fig. 41).

Indeed in this situation, the highest and lowest slope have the same absolute value, $\left|i_{b}\left(\mathrm{~T}_{p} / 4\right)\right|=\left|i_{b}\left(3 \mathrm{~T}_{p} / 4\right)\right|=$ 0.7 . Accordingly, in these configurations the plasma frequencies take on values $\left(\omega_{P}^{0.0}\left(\mathrm{~T}_{p} / 4\right)=\omega_{P}^{0.0}\left(3 \mathrm{~T}_{p} / 4\right) \simeq\right.$ 0.85 ), very close to that for which the RA minimum is observed. The suppression of the dynamic RA, as the noise intensity increases, is evident in the curves obtained for $i_{0}=0.1$. In particular, the stochastic RA emerges at $\gamma_{s R A}^{0.1} \simeq 0.005$, with the minimum located in $\omega_{s R A}^{0.1} \simeq 0.7$. In these conditions the dynamics is exclusively ruled by the noise fluctuations which "do not see" the potential details. Using these small values of bias current, a trapping phenomenon occurs for $\omega \geq 1$. This effect is due to the inability of the particle to leave a minimum, since the frequency of the oscillating potential is larger than the characteristic frequency of the well, i.e. the plasma frequency. These trapping phenomena however disappear for higher values of the noise intensity. For $i_{0}=0.5,0.9$ the potential is tilted enough to become, in the lowest configuration, well-free. If $i_{0}=0.5$ the double-minimum dynamic RA is still present around the frequencies $\omega_{d R A}^{0.5} \simeq 0.72,1.02$, but the MFPT value in the first RA minimum is smaller than that calculated for $i_{0}=0.1$. This is due to the fact that for $i_{0}=0.5$ the virtual particle, i.e. the phase difference between the wave functions of the two superconductors, is able to leave the potential well in a shorter time, escaping through the right potential barrier. The slope $i_{0}=0.5$ in fact is sufficient to produce a right-side escape event already after a quarter of an oscillation period (indeed $\tau \simeq \mathrm{T}_{p} / 4$ ), whereas for $i_{0}=0.1$ the particle needs one complete oscillation to pass the same barrier. On the other hand, the values of $\tau$ in the second RA valley for $i_{0}=0.1$ and $i_{0}=0.5$ are almost equal, since the particle needs more than one complete oscillation (for both slopes $\left.\tau \simeq \mathrm{T}_{p}+3 \mathrm{~T}_{p} / 4\right)$ to escape from the left potential barrier. Setting $i_{0}=0.9$, the dynamic RA is just hinted and only the minimum around $\omega_{d R A}^{0.9} \simeq 1.6$, corresponding to a highly sloping potential, is clearly detectable. Trapping phenomena at high frequencies are still present. Specifically they appear for frequencies larger than the following threshold values: $\omega_{t h r}^{0.5} \simeq 1.2$ and $\omega_{t h r}^{0.9} \simeq 2.4$. Increasing the value of the bias current, the right potential barrier decreases. As a consequence, trapping phenomena can occur only if the potential oscillates at higher frequencies. Furthermore, the parabolic approximation (linearization of the potential at the bottom of the well) used to calculate the plasma frequency (see Eq. (40) fails for a highly tilted potential. In Fig. [3 we show the behaviour of the MFPT as a function of the noise intensity $\gamma$. In all panels of Fig. 3 we note the presence of another noise induced effect, known as noise enhanced stability (NES)33,54,65-78. Indeed the curves of $\tau$ vs $\gamma$ are characterized by a nonmonotonic behavior with the presence of a maximum. This nonmonotonic behavior is different from that expected from the Kramers theory and its extensions $\underline{79}-81$. The enhancement of stability present in the curves of Fig. 3, first noted by Hirsch et al. $\frac{82}{}$, has been observed in different physical and biological systems, and belongs to a highly topical interdisciplinary research field, ranging from condensed matter physics to molecular biology and cancer growth dynamics ${ }^{71,83}$. More in detail, we note that the $\tau$ vs $\gamma$ behaviour shows the presence of NES for any frequency taken in an interval around the different frequencies $\omega_{d R A}$. This suggests that the origin of this nonmonotonic effect can lie in the resonance phenomenon, involving the plasma frequency, previously discussed about the RA effect. Specifically, for $i_{0}=0.0$ this effect occurs for $\omega_{N E S} \in[0.43-0.87]$. For each value $i_{0}=0.1,0.5$ of the bias current, there are two $\omega_{d R A}$ frequencies and, correspondingly, two different ranges of frequencies giving evidence of NES effects.

In detail: $\omega_{N E S}^{(1)} \in[0.42-0.78]$ and $\omega_{N E S}^{(2)} \in[0.84-1.02]$ for $i_{0}=0.1$, and $\omega_{N E S}^{(1)} \in[0.24-0.77]$ and $\omega_{N E S}^{(2)} \in$ $[0.97-1.14]$ for $i_{0}=0.5$.

Using highly tilted potential, i.e. $i_{0}=0.9$, there is only one RA minimum and, according to the correspondence previously observed, only one range of frequencies $\left(\omega_{N E S} \in[0.4-2.4]\right)$ for which the NES phenomenon is found. According to this analysis, the curves of Fig. 5 , obtained for different values of the noise correlation time $\left(\tau_{c}=0.0,1.0,5,10,\right)$ show the presence of NES for values of $\omega$ chosen in the intervals given above. In all curves, 

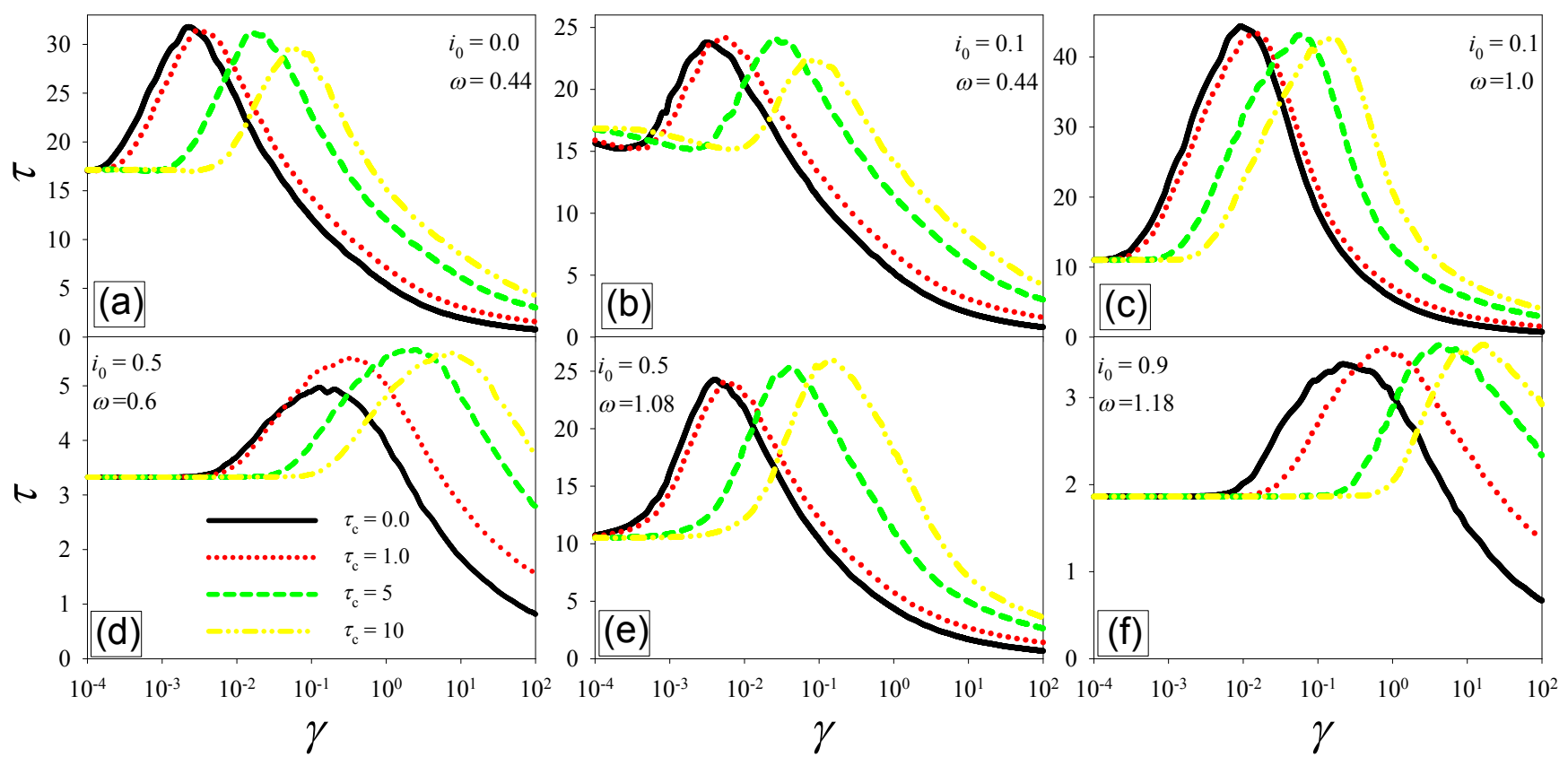

FIG. 5. (Color online) MFPT as a function of $\gamma$, for different values of $\omega, i_{0}$ and $\tau_{c}$. In detail: (a) $i_{0}=0, \omega=0.44$; (b) $i_{0}=0.1$, $\omega=0.44 ;$ (c) $i_{0}=0.1, \omega=1.0 ;$ (d) $i_{0}=0.5$ and $\omega=0.6$; (e) $i_{0}=0.5, \omega=1.08$; (f) $i_{0}=0.9, \omega=1.18$. The legend in panel d refers to all pictures.

as $\tau_{c}$ increases, the maxima are shifted towards higher values of the noise intensity. Moreover, the MFPT values around the NES maxima tend to slightly reduce for low slopes (small values of $i_{0}$ ) of the oscillating potential (panels a, b and c of Fig. 5) and to increase for high slopes (panels d, e and $f$ of the Fig. 5). These features,

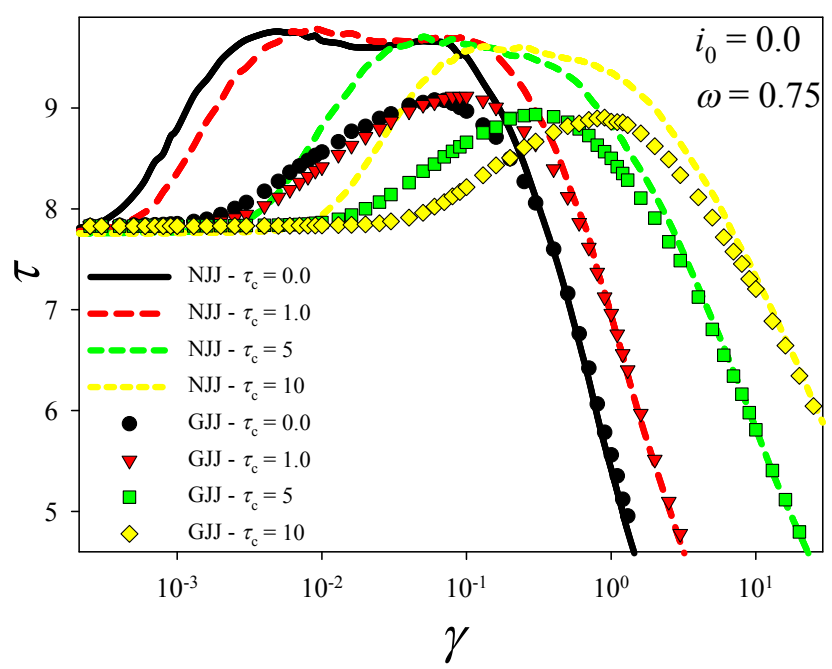

FIG. 6. (Color online) MFPT as a function of $\gamma$, for $\omega=$ $0.75, i_{0}=0.0$, and different values the noise correlation time: $\tau_{c}=0.0,1.0,5,10$. Lines and symbols represent results for a normal JJ (NJJ) and a graphene-based JJ (GJJ), respectively. i.e. the shift towards higher frequencies and modification in the maxima of MFPT for increasing values of $\tau_{c}$, are present also in a conventional JJ. In Fig. 6] where $i_{0}=0.0$ and $\omega=0.75$, it is possible to observe that for a normal JJ respect to a graphene junction: i) the NES maxima are broadener; ii) the phase particle remains confined in the potential well for longer time, i.e. the $\tau$ values are slightly higher (in accordance with the results of Fig. (4); iii) the NES effect appears for lower noise intensities. Conversely, the behaviors of normal and graphene JJs coincide for larger values of the noise intensity $\gamma$, since the specific potential profile becomes irrelevant due to the strength of random fluctuations.

\section{PROBABILITY DENSITY FUNCTIONS}

To deeply understand the behavior of the MFPT, we expand further the theoretical analysis discussing the PDFs of the switching times $P\left(t^{e s c}\right)$. We set the parameters of system and noise source to obtain nonmonotonic effects in the MFPT data. Every PDF is costructed implementing $N_{\exp }=10^{7}$ experiments, and every curve is normalized to unity. Whenever possible, the time $t$ will be normalized to the washboard oscillation period $\mathrm{T}_{p}$, to couple the switching dynamics with the inclinations assumed by the potential. The panels (a), (b), (c) and (d) of the Fig. 7 show $P\left(t^{e s c}\right)$ in function of time $t$, by changing the initial bias current values $i_{0}=\{(\mathrm{a})$ 0 , (b) 0.1, (c) 0.5 , (d) 0.9$\}$. These data allow to explore the switching dynamics in correspondence of pe- 


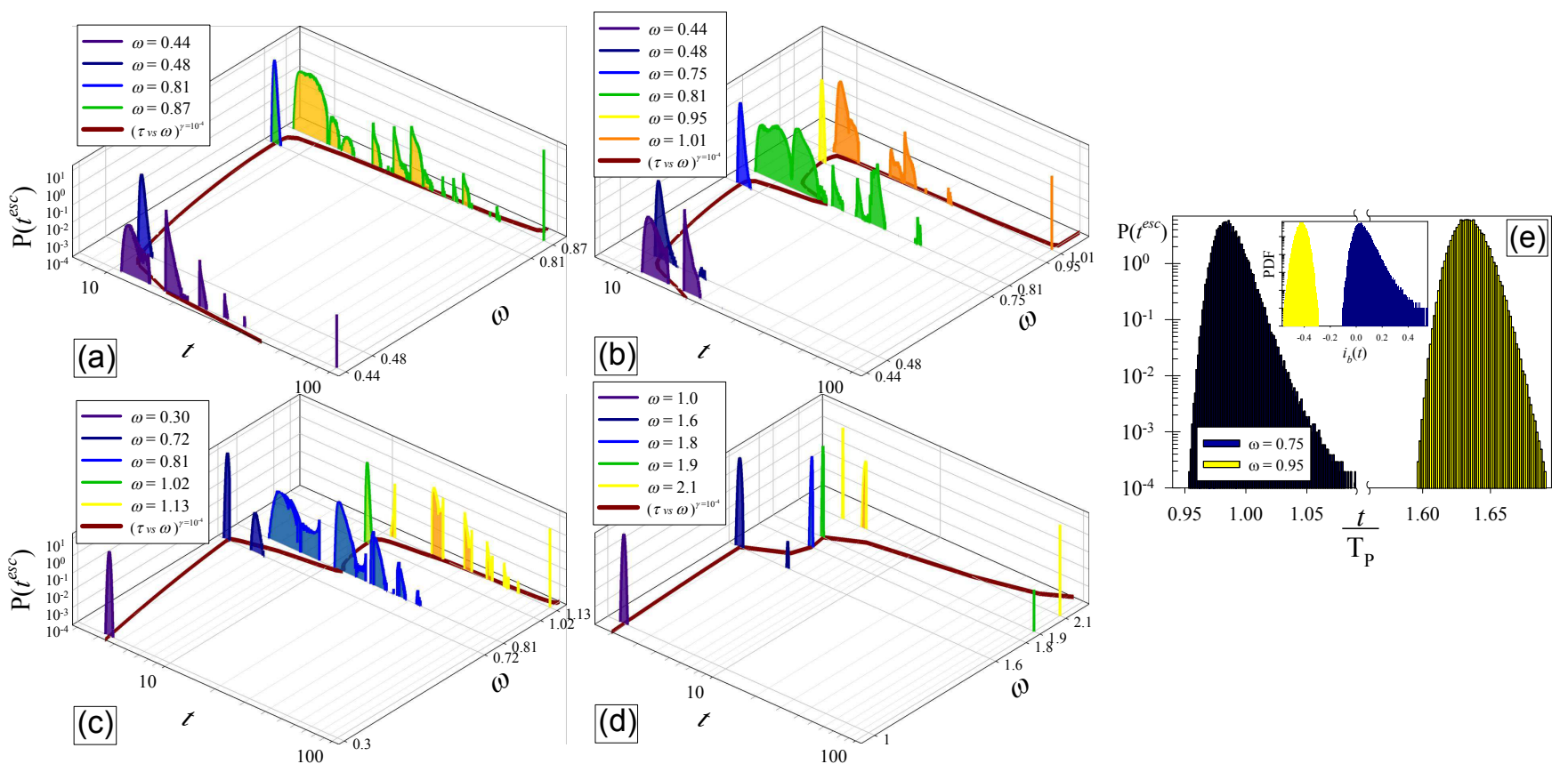

FIG. 7. (Color online) Panels (a), (b), (c) and (d): PDFs as a function of the time $t$, varying $\omega$. Every picture is obatined fixing the values of $\gamma=10^{-4}, \tau_{c}=0$ and $i_{0}=\left\{(\mathrm{a}) i_{0}=0,(\mathrm{~b}) i_{0}=0.1,(\mathrm{c}) i_{0}=0.5,(\mathrm{~d}) i_{0}=0.9\right\}$. The MFPT versus $\omega$ curves corresponding to the dynamic RA effects (see solid lines in Fig. 4) are also shown. The PDF and $t$ axes are logarithmic. Panel (e): Semi-log plot of the PDFs as a function of the time $t$, normalized to the washboard oscillation period $\mathrm{T}_{p}$, setting $i_{0}=0.1$ and $\omega=\omega_{d R A}^{0.1}=\{0.75,0.95\}$. The inset shows the same PDF data in function of the bias current $i_{b}(t)$.

culiar points of $\tau$ vs $\omega$ curves (see solid lines lying on the $t-\omega$ planes and in Fig. (4), calculated for $\gamma=10^{-4}$. Setting $\omega=\omega_{d R A}$, the resonance-like dynamics results in single-peack PDFs, centered around the MFPT values. This suggests that, in all the experiments, the phase particles tend to follow almost the same trajectory to escape from the initial metastable state. In particular, the panel (e) of Fig. 7 shows the PDF calculated selecting $\omega=\omega_{d R A}^{0.1}=\{0.75,0.95\}$, as a function of the normalized time $t / T_{p}$. As already noted, setting $\omega=0.75$, that is in the first dynamic RA minimum, the particle tends to escape through the right barrier after almost one oscillation of the washboard potential, instead setting $\omega=0.95$, that is in the second dynamic RA minimum, the left-side escapes occur when $t \simeq 1.6 \mathrm{~T}_{p}$. These peacks show asymmetry and long tails. The asymmetry is more pronunced in low frequency data, due to the time that the washboard spend in the configurations supporting the escape events, that increase reducing its oscillation frequency. The insets in the panel (e) of Fig. 7 show the PDFs plotted in function of the bias current $i_{b}(t)$, to compare, at least qualitatively, these data with the switching current probability $P\left(I_{c}\right)$, often studyed in the JJ framework. The shape of these single peack PDFs recall the termally activated switching current distribution in SGS systems (see Ref. 18 and 21), and their orientation depends to the washboard dynamics when the escape event occurs. The PDFs for frequencies within the dynamic RA minima are formed by single peacks, but tend to broaden moving from $\omega=\omega_{d R A}$ (see panels (a) and (b) of the Fig. 7). Far from these frequencies, that is when the $\tau$ values grow, the PDFs show multi-peacks structures, suggesting that the trajecotires followed by the phase particle in the various experiments get very spread. This occurs also for $\omega=0.81$ and $i_{0}=\{0.1,0.5\}$, that is in correspondence of the narrow maximum "intra-RA minima" of $\tau$ data (see the panels (b) and (c)). The PDFs for high frequencies show high narrow peacks for $t=t_{\max }$ indicating the particle inability to leave the metastable state, i.e. trapping events. In particular, for $i_{0}=0$ and $\omega=0.87$ almost $21 \%$ of the experiments give entrapment, whereas for $i_{0}=0.1$ and $\omega=1.01$ this occurs in $\sim 2 \%$ of the experiments. Increasing $i_{0}$, the width of the peacks reduces, but the multi-peacks structures in high frequencies PDF are still evident as well as the trapping phenomena. In detail, selecting $i_{0}=5$ and $\omega=1.13$ the probability that the phase particle undergoes a trapping is $\sim 4 \%$, and for $i_{0}=9$ and $\omega=2.1$ is $\sim 23 \%$. The panels $(\mathrm{a}-\mathrm{f})$ of the Fig. 8 show $P\left(t^{e s c}\right)$ in function of normalized time $t / \mathrm{T}_{p}$ obtained setting system and noise parameters in analogy with those setted in the panels $(\mathrm{a}-\mathrm{f})$ of the Fig. 5 , that is: panel (a) $i_{0}=0, \omega=0.44$, panel (b) $i_{0}=0.1$, $\omega=0.44$, panel (c) $i_{0}=0.1, \omega=1.0$, panel (d) $i_{0}=0.5$ and $\omega=0.6$, panel (e) $i_{0}=0.5, \omega=1.08$ and panel (f) $i_{0}=0.9, \omega=1.18$. These PDF data allow to explore the NES effects described in the Fig. [5 (solid curves reported on the $t-\omega$ planes of the panels of Fig. 8). The creation of NES maxima is due to the possibility that random fluc- 


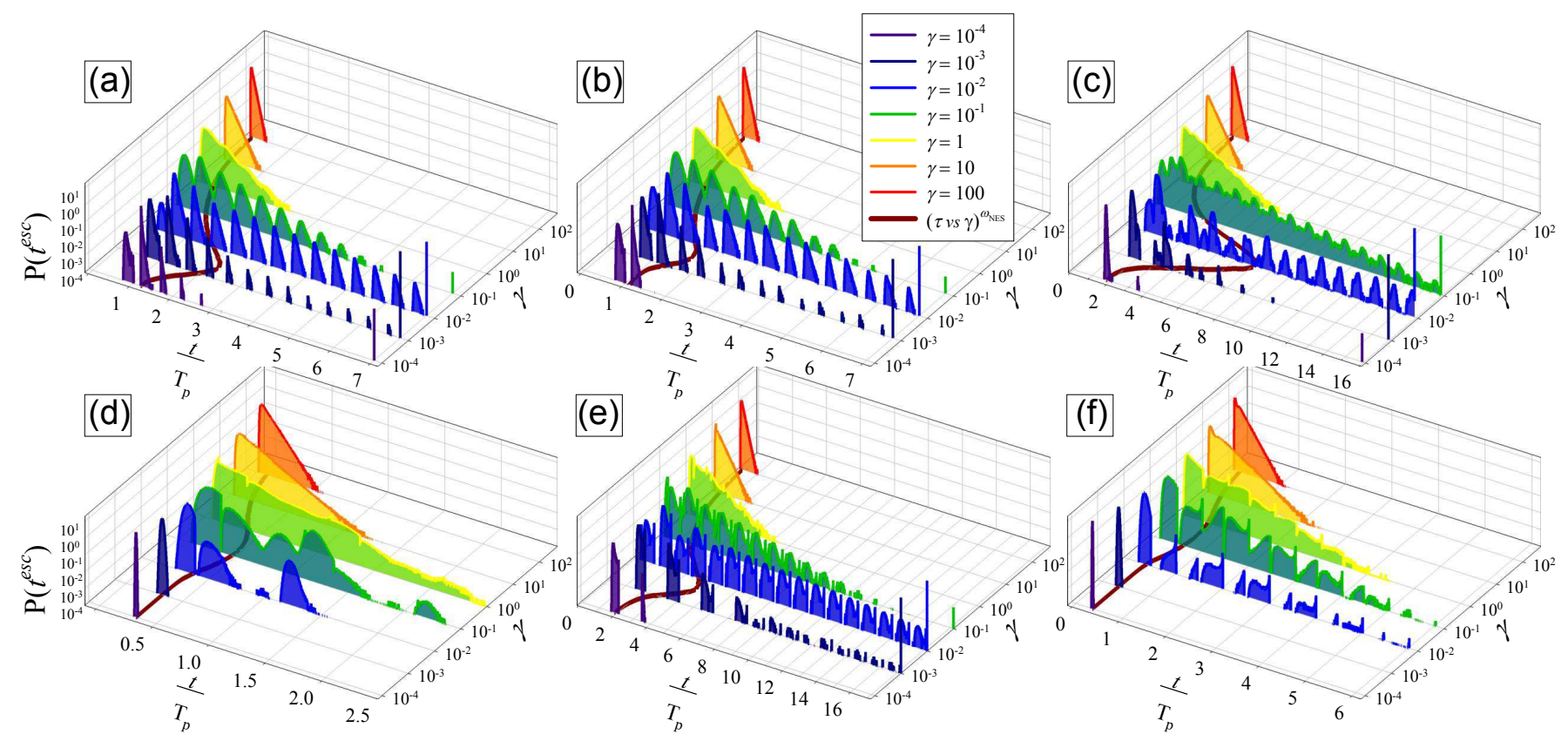

FIG. 8. (Color online) PDF as a function of the time $t$, normalized to the washboard oscillation period $\mathrm{T}_{p}$, varying $\gamma$. Every picture is obatined fixing the values of $\omega, i_{0}$ and $\tau_{c}=0$. In detail: (a) $i_{0}=0, \omega=0.44$; (b) $i_{0}=0.1, \omega=0.44$; (c) $i_{0}=0.1$, $\omega=1.0 ;$ (d) $i_{0}=0.5$ and $\omega=0.6$; (e) $i_{0}=0.5, \omega=1.08$; (f) $i_{0}=0.9, \omega=1.18$. Every picture shows also the MFPT versus $\gamma$ curve corresponding to NES effect (see solid lines in Fig. 5 obtained using the same values for the other parameters. The PDF and $\gamma$ axes are logarithmic. The legend in panel (b) refers to all pictures.

tuations confine the particle inside the well also in the potential configurations good for escape events. In correspondence of the NES maxima, the PDF are composed by long regular sequences of peacks, with amplitude esponentially decreasing in time. For low potential inclination (see panels $(\mathrm{a}-\mathrm{c})$ ) and $\gamma \leq 10^{-1}$ these sequences are formed by two peacks per period, corresponding to a right- and left-side escapes, and every peacks have an almost triangular shape. Increasing the noise amplitude, the peacks tend to get less high but more large, melting in an almost trinagular large peacks for $\gamma \geq 1$. The low frequency PDF (panels (a), (b) and (d)) show peacks spreading over almost 2, 1 and 0.5 periods $\mathrm{T}_{p}$ for $\gamma=1$, 10 and 100 respectively. Looking high frequencies PDF (panels (c), (e) and (f)) the width of these peack is twice, that is they spread over almost 4,2 and 1 periods $\mathrm{T}_{p}$ for $\gamma=1,10$ and 100 respectively. The PDF in the panel (d) doesn't show regularity, the potential is highly tilted and its frequency is not enough to generate long-living trajectories. The panels (e) and (f) regard highly tilted potentials too, but the frequency is high enough to trap the phase particle inside the initial well for long time generating interesting periodic peacks structures. Every period contains two asymmetric and very close peacks, a first intense and large due to right-side escapes and a second narrow connected with the left-side escapes.

\section{CONCLUSIONS}

We explored the influence of thermal fluctuations on the behavior of a ballistic graphene-based Josephson junction in the short-junction regime. In particular, we analyzed how random fluctuations affect the lifetime of the superconductive state in an underdamped currentbiased JJ. The analysis was performed within the framework of the resistively and capacitively shunted junction (RCSJ) model, using a proper non-sinusoidal currentphase relation, characteristic of graphene. Specifically we investigated the mean first passage time (MFPT) of the phase particle, i.e. the phase difference across the junction, initially placed in a minimum of the tilted washboard-like potential. In particular, we studied the MFPT as a function of different parameters of the system and external perturbations, i.e. Gaussianly distributed random fluctuations and periodical driving signal. We found nonmonotonic behavior of the lifetime, $\tau$, of the superconductive state as a function of the noise intensity $\gamma$, driving frequency $\omega$ and fixing the initial value of the bias current $i_{0}$. These results indicate the presence of noise induced phenomena, such as stochastic resonant activation (RA) and noise enhanced stability (NES) with different features, strongly depending on the initial value, $i_{0}$, of the bias current. In particular, we observed ranges of parameters in which MFPT show evidence of dynamic and stochastic RA, including a multi-minimum RA effect in the low-noise-intensity regime. Finally, we observed 
changes in the behaviour of MFPT, when the white noise source is replaced by a coloured noise source with different values of the correlation time $\tau_{c}$.

Our study provides information on the role played by random (both thermal and correlated) fluctuations in the switching dynamics from the superconductive state to the resistive one of a graphene-based JJ. The results obtained can help to better understand the role of fluctuations in the electrodynamics of new generation graphene-based superconductive devices, such as Josephson junctions, Josephson sensors, dc-SQUIDs and gate-tunable phase qubits, contributing to improve their performances.
In conclusion this work, which is well placed in the framework of the nonequilibrium statistical mechanics, due to the presence of an emerging material, such as graphene, with unique electrical properties, presents relevant and interesting results from several points of view.

\section{ACKNOWLEDGEMENTS}

Authors acknowledge the financial support of Ministry of Education, University, and Research of Italian Government (MIUR). They also thank Dr. I. Hagymási for fruitful discussions and useful suggestions.
${ }^{1}$ K. Novoselov, A. Geim, S. Morozov, D. Jiang, Y. Zhang, S. Dubonos, I. Grigorieva, and A. Firsov, Science 306, 666 (2004).

${ }^{2}$ H. Heersche, P. Jarillo-Herrero, J. Oostinga, L. Vandersypen, and A. Morpurgo, Nature 446, 56 (2007).

3 X. Du, I. Skachko, and E. Andrei, Phys. Rev. B 77, 184507 (2008).

4 B. Uchoa and A. Castro Neto, Phys. Rev. Lett. 98, 146801 (2007).

5 A. Fedorov, N. Verbitskiy, D. Haberer, C. Struzzi, L. Petaccia, D. Usachov, O. Vilkov, D. Vyalikh, J. Fink, M. Knupfer, B. Buchner, and A. Gruneis, Nat. Commun. 5, 3257 (2014).

${ }^{6}$ C. Girit, V. Bouchiat, O. Naaman, Y. Zhang, M. Crommie, A. Zettl, and I. Siddiqi, Nano Letters 9, 198 (2009).

7 C. Girit, V. Bouchiat, O. Naaman, Y. Zhang, M. Crommie, A. Zettl, and I. Siddiqi, Phys. Status Solidi B 246, 2568 (2009).

8 J. Voutilainen, M. Laakso, and T. Heikkil, J. Appl. Phys. 107, 064508 (2010).

9 X. Du, D. E. Prober, H. Vora, and C. B. Mckitterick, Graphene 2D Mater 1, 1 (2014).

10 A. Castro-Neto, F. Guinea, N. Peres, K. Novoselov, and A. Geim, Rev. Mod. Phys. 81, 109 (2009).

11 C. Beennaker, Phys. Rev. Lett. 97, 067007 (2006).

12 A. Titov, M. Ossipov and C. Beennaker, Phys. Rev. B 75 , 045417 (2007).

13 M. Maiti and K. Sengupta, Phys. Rev. B 76, 054513 (2007).

14 M. Titov and C. Beennaker, Phys. Rev. B 74, 041401 (2006), (R).

15 J. Lambert, A. Carabello, and R. Ramos, IEEE Trans. Appl. Supercon. 4, 2716 (2011).

16 F. Miao, W. Bao, H. Zhang, and C. Lau, Solid State Commun. 149, 1046 (2009).

17 D. Jeong, J. Choi, G. Lee, S. Jo, Y. Doh, and H. Lee, Phys. Rev. B 83, 094503 (2011).

18 U. Coskun, M. Brenner, T. Hymel, V. Vakaryuk, A. Levchenko, and A. Bezryadin, Phys. Rev. Lett. 108, 097003 (2012).

19 I. Hagymási, A. Kormányos, and J. Cserti, Phys. Rev. B 82, 134516 (2010).

20 N. Mizuno, B. Nielsen, and X. Du, Nature Comm. 21, 734 (2013).
21 G. Lee, D. Jeong, J. Choi, Y. Doh, and H. Lee, Phys. Rev. Lett. 107, 146605 (2011).

22 J. Choi, G. Lee, S. Park, D. Jeong, J. Lee, H. Sim, Y. Doh, and H. Lee, Nature Comm. , 2525 (2013).

23 A. Barone and G. Paternò, Physics and Applications of the Josephson Effect (1982).

24 A. Pankratov and B. Spagnolo, Phys. Rev. Lett. 93, 177001 (2004).

25 A. Gordeeva and A. Pankratov, Appl. Phys. Lett. 88, 022505 (2006).

26 A. Gordeeva and A. Pankratov, J. Appl. Phys. 103, 103913 (2008).

27 G. Augello, D. Valenti, and B. Spagnolo, Int. J. Quantum Inf. 6, 801 (2008).

28 A. Gordeeva, A. Pankratov, and B. Spagnolo, Int. J. Bif. Chaos 18, 2825 (2008).

${ }^{29}$ K. Fedorov and A. Pankratov, Phys. Rev. B 76, 024504 (2007).

30 K. Fedorov, A. Pankratov, and B. Spagnolo, Int. J. Bif. Chaos 18, 2857 (2008).

31 K. Fedorov and A. Pankratov, Phys. Rev. Lett. 103, 260601 (2009).

32 G. Augello, D. Valenti, A. Pankratov, and B. Spagnolo, Eur. Phys. J. B 70, 145 (2009).

33 D. Valenti, C. Guarcello, and B. Spagnolo, Phys. Rev. B 89, 214510 (2014).

34 G. Augello, D. Valenti, and B. Spagnolo, Eur. Phys. J. B 78, 225 (2010).

35 Y. Yu and S. Han, Phys. Rev. Lett. 91, 1270031 (2003).

36 G. Sun, N. Dong, G. Mao, J. Chen, W. Xu, Z. Ji, L. Kang, P. Wu, Y. Yu, and D. Xing, Phys. Rev. E 75, 021107 (2007).

37 C. Pan, X. Tan, Y. Yu, G. Sun, L. Kang, W. Xu, J. Chen, and P. Wu, Phys. Rev. E 79, R030104 (2009).

38 H. Grabert, Phys. Rev. B 77 (2008).

39 D. Urban and H. Grabert, Phys. Rev. B 79, 113102 (2009).

40 J. Ankerhold, Physical Review Letters 98 (2007).

41 E. Sukhorukov and A. Jordan, Physical Review Letters 98 (2007).

42 M. Köpke and J. Ankerhold, New Journal of Physics 15 (2013)

43 G. Filatrella and V. Pierro, Phys. Rev. E 82 (2010).

44 P. Addesso, G. Filatrella, and V. Pierro, Phys. Rev. E 85 (2012). 
45 P. G. De Gennes, Superconductivity of Metals and Alloys (Benjamin, New York, 1966).

46 P. Brouwer and C. Beenakker, Chaos, Solitons Fractals 8, 1249 (1997).

47 J. Tworzydło, B. Trauzettel, M. Titov, A. Rycerz, and C. W. J. Beenakker, Phys. Rev. Lett. 96, 246802 (2006).

48 I. Hagymási, (private communication) (2014), the Fig.1(a) of the Ref. 19 shows data only for $T / T_{c}=0,0.53,0.71$. The proof about the temperature-indipendent behavior of $i(\varphi)$ for $T / T_{c} \lesssim 0.25$ is the result of private communications with the author.

49 H. Grabert and U. Weiss, Phys. Rev. Lett. 53, 1787 (1984).

50 C. W. Gardiner, Handbook of Stochastic Methods (Springer-Verlag, Berlin, 2004) p. 106.

51 C. English, D. Hamilton, C. Chialvo, I. Moraru, N. Mason, and D. V. Harlingen, arXiv:1305.0327 (2013).

52 C. Doering and J. Gadoua, Phys. Rev. Lett. 69, 2318 (1992).

53 R. Mantegna and B. Spagnolo, Phys. Rev. Lett. 84, 3025 (2000).

54 A. Dubkov, N. Agudov, and B. Spagnolo, Phys. Rev. E 69 (2004).

${ }^{55}$ R. Mantegna and B. Spagnolo, J. Phys. IV (France) 8, Pr6 (1998).

56 P. Pechukas and P. Hänggi, Physical Review Letters 73, 2772 (1994).

57 M. Marchi, F. Marchesoni, Gammaitoni, E. MenichellaSaetta, and S. Santucci, Phys. Rev. E 54, 3479 (1996).

58 B. Dybiec and E. Gudowska-Nowak, J. Stat. Mech.: Theory Exp. , P05004 (2009).

59 S. Miyamoto, K. Nishiguchi, Y. Ono, K. Itoh, and A. Fujiwara, Phys. Rev. B 82 (2010).

60 Y. Hasegawa and M. Arita, Physics Letters A 375, 3450 (2011).

61 A. Fiasconaro and B. Spagnolo, Phys. Rev. E 83 (2011).

${ }^{62}$ M. Devoret, J. Martinis, D. Esteve, and J. Clarke, Phys. Rev. Lett. 53, 1260 (1984).
63 M. Devoret, J. Martinis, and J. Clarke, Phys. Rev. Lett. 55, 1908 (1985).

64 J. Martinis, M. Devoret, and J. Clarke, Phys. Rev. B 35, 4682 (1987).

65 R. Mantegna and B. Spagnolo, Phys. Rev. Letters 76, 563 (1996).

66 N. Agudov and B. Spagnolo, Phys. Rev. E 64, 351021 (2001).

67 B. Spagnolo, N. Agudov, and A. Dubkov, Acta Phys. Pol. B 35, 1419 (2004).

68 P. D'Odorico, F. Laio, and L. Ridolfi, Proc. Natl. Acad. Sci. U.S.A. 102, 10819 (2005).

69 A. Fiasconaro, B. Spagnolo, and S. Boccaletti, Phys. Rev. E 72 (2005).

70 P. Hurtado, J. Marro, and P. Garrido, Phys. Rev. E 74 (2006).

71 B. Spagnolo, A. Dubkov, A. Pankratov, E. Pankratova, A. Fiasconaro, and A. Ochab-Marcinek, Acta Phys. Pol. B 38, 1925 (2007).

72 R. Mankin, E. Soika, A. Sauga, and A. Ainsaar, Phys. Rev. E - Statistical, Nonlinear, and Soft Matter Physics $\mathbf{7 7}$ (2008).

73 M. Yoshimoto, H. Shirahama, and S. Kurosawa, J. Chem. Phys. 129 (2008).

74 A. Fiasconaro and B. Spagnolo, Phys. Rev. E 80 (2009).

75 M. Trapanese, J. Appl. Phys. 105, 07 D313 (2009).

76 A. Fiasconaro, J. Mazo, and B. Spagnolo, Phys. Rev. E 82 (2010).

77 J. Li and J. Euczka, Phys. Rev. E 82 (2010).

78 A. Smirnov and A. Pankratov, Phys. Rev. B 82 (2010).

${ }^{79}$ H. Kramers, Physica 7, 284 (1940).

80 V. Mel'Nikov, Phys. Rep. 209 (1991).

81 P. Hänggi, P. Talkner, and M. Borkovec, Reviews of Modern Physics 62, 251 (1990).

82 J. Hirsch, B. Huberman, and D. Scalapino, Physical Review A 25, 519 (1982).

83 B. Spagnolo, P. Caldara, A. La Cognata, G. Augello, D. Valenti, A. Fiasconaro, A. Dubkov, and G. Falci, Acta Physica Polonica B 43, 1169 (2012). 